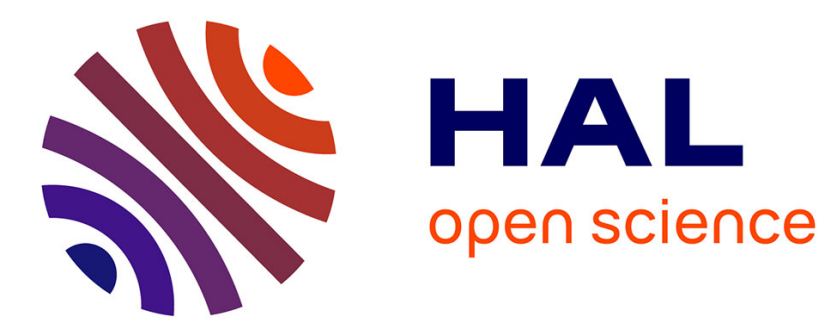

\title{
Etalonnage des intensimètres. Applications industrielles
}

J. Wasmer

\section{To cite this version:}

J. Wasmer. Etalonnage des intensimètres. Applications industrielles. Journal de Physique IV Proceedings, 1994, 04 (C5), pp.C5-255-C5-258. 10.1051/jp4:1994551 . jpa-00253046

\section{HAL Id: jpa-00253046 https://hal.science/jpa-00253046}

Submitted on 1 Jan 1994

HAL is a multi-disciplinary open access archive for the deposit and dissemination of scientific research documents, whether they are published or not. The documents may come from teaching and research institutions in France or abroad, or from public or private research centers.
L'archive ouverte pluridisciplinaire HAL, est destinée au dépôt et à la diffusion de documents scientifiques de niveau recherche, publiés ou non, émanant des établissements d'enseignement et de recherche français ou étrangers, des laboratoires publics ou privés. 


\title{
Etalonnage des intensimètres. Applications industrielles
}

\author{
J. WASMER
}

Société AKSUD, 11 Allée des Imprimeurs, Z.I. Secteur A, 06700 Saint Laurent du Var, France

\begin{abstract}
:
The determination of Sound power levels of machines using acoustic Intensity techniques is an interesting method. To obtain a high accuracy, it is necessary to reduce the phase mismatch of the microphones. The usual solutions are physical matching of the microphones (matched probe) or the use of calibration procedures to eliminate the phase mismatch of the two channels. Theoritically, using a good matching probe, acousticians do not need to use calibration procedures. However, at industrial sites, measurement systems were usually submitted in heavy conditions (environments, transport, etc.... It is therefore necessary to check their quality before use.

AKSUD and CETIM have developped a tested and easily used system : special, matched intensity probe and a on-site calibrator. The principal advantage of this system is based on the simplicity procedures. In fact, in the classical calibration method, operators need to take the microphones off the probe assembly during the use of electrostatic excitation components. With these new products, the probes are built with an electrostatic component. The calibration will be processed without removing the microphones from the assembly. In this way, the calibration procedures are easier to be repeated more frequently.

Today, with this new system (probe and on-site calibrator), acousticians have an efficient means of control of probe quality to ensure good measurement results.
\end{abstract}

\section{INTRODUCTION}

A l'heure où la certification ISO 9000 prend essor dans les entreprises industrielles, les services de contrôle et d'essais doivent se préparer à s'adapter à de nouvelles manières de travail plus rigoureuses et plus méthodiques.

Dans les laboratoires de mesures, les acousticiens doivent maîtriser l'assurance - qualité de leurs mesures selon des procédures bien établies. Or, puisque toute mesure physique comporte des incertitudes, la technique de l'intensimétrie acoustique n'échappe pas à cette règle et comporte un certain nombre de sources d'erreurs qui sont liées aux techniques de traitement de signal, aux algorithmes de calcul, à l'opérateur et surtout aux capteurs.

Les principales sources d'erreurs qui affectent la précision des mesures d'intensimétrie ont été étudiées et publiées par différents auteurs [1], [2], [3], [4].

Dans une démarche d'amélioration de la qualité des mesures, concernant la sonde et l'étalonnage, la Société AKSUD et le CETIM ont développé et testé un système simple d'emploi, afin que l'utilisateur dispose de moyens lui permettant de s'assurer la validité de ses mesures : il s'agit de l'association d'une sonde appariée en phase par sa conception, dotée de moyens permettant le contrôle de validation des mesures sur site, et d'un calibreur conçu, aussi bien pour. le contrôle d'étalonnage sur site ou en atelier, que pour la maintenance préventive de l'intensimètre.

Le principal avantage de ce système résulte dans la simplicité de mise en oeuvre des procédures de tests et d'étalonnage, ce qui permet à l'utilisateur de répéter plus fréquemment les opérations de validation avant les séries de mesures. 


\section{PROBLEMES POSES PAR LES MESURES D'INTENSIMETRIE}

L'intensimétrie acoustique est de loin la technique la plus intéressante et la mieux adaptée à la détermination de la puissance acoustique, ainsi qu'à l'analyse du comportement d'une source de bruit.

Cependant, elle demande de la connaissance, du savoir faire et de l'expérience pour parvenir à des résultats cohérents.

Les sources d'erreurs sont nombreuses et parfois interdépendantes.

On peut les classer en trois catégories selon le schéma suivant :

1) La méthodologie qui concerne les problèmes liès aux algorithmes de calcul et au traitement de signal, comme c'est le cas par exemple de l'approximation par différences finies.

2) L'instrumentation qui comporte les capteurs, l'électronique de conditionnement du signal (acquisition, transmission, filtrage) et le système de traitement.

3) L'environnement déterminé par la nature du champ sonore et par le savoir faire de l'opérateur.

L'environnement n'est pas à proprement parler une source d'erreur, mais la connaissance de son incidence permet de déterminer les approximations de ce paramètre, compatibles avec la précision finale que l'on peut atteindre en tenant compte de l'ensemble des sources d'erreurs.

Parmi les sources d'erreurs évoquées précédemment, celles qui proviennent des capteurs sont généralement prédominantes, particulièrement l'erreur dûe au déphasage relatif entre les deux voies de mesure.

\section{LA QUALITE DES SONDES}

Une sonde unidimensionnelle d'intensimétrie acoustique comporte deux voies de mesure et sa qualité dépend principalement de sa réponse en phase. Plusieurs techniques ont été proposées pour maîtriser ce paramètre [1], [5], [6].

Or, comme les capteurs contenus dans la sonde sont à l'origine des principales distorsions de phase, il a semblé judicieux d'analyser les différentes techniques éprouvées en vue de s'assurer la validité de celles-ci dans le cadre d'applications industrielles et d'en apprécier les contraintes.

Nous examinerons donc à cette fin les différentes techniques envisageables.

$\left.1^{\circ}\right)$ Sonde constituée de microphones appariés physiquement.

Comme c'est dans le domaine des fréquences les plus basses que les erreurs de phase prédominent en raison de la faible distance de séparation des microphones vis-à-vis des longueurs d'ondes des signaux acoustiques à traiter, c'est aussi dans cette région du spectre que les microphones doivent être appariés avec le plus grand soin.

En règle générale, la différence de phase entre les deux voies microphoniques sera d'autant plus faible que les fréquences de coupure inférieure seront, à la fois les plus proches possible et les plus basses.

L'expérience a cependant prouvé qu'avec le temps, des microphones initialement appariés par construction finissent par dériver l'un par rapport à l'autre et ne conservent plus les performances requises par les normes de mesure d'intensimétrie.

$2^{\circ}$ ) Sonde associée à une correction de phase par logiciel.

Un tel logiciel présente l'avantage qu'il permet d'employer une sonde d'intensimétrie équipée de microphones non appariés en phase. II est alors nécessaire d'étalonner l'intensimètre à l'aide d'un calibreur d'intensimétrie. Cette opération n'est pas contraignante pour l'utilisateur dans la mesure où elle a lieu lors des contrôles en fin de production .

Toutefois, les mêmes incertitudes que dans le cas d'une sonde appariée par construction pourront apparaître dans le temps si les microphones dérivent inégalement en phase.

Toutefois, des contrôles périodiques permettent de minimiser l'incidence de ces dérives. 
$3^{\circ}$ ) Sonde équipée de microphones à cavités multiples.

La réponse en phase de ces microphones est parfaitement maîtrisée dans la gamme des fréquences les plus basses de l'intensimétrie acoustique [5]. De plus, les dérives dans le temps demeurent très faibles, de sorte qu'il n'est pas nécessaire de réétalonner fréquemment les intensimètres équipés de telles sondes.

Cependant, des microperforations dans les membranes des microphones peuvent se produire dans le temps par suite de la corrosion et devenir le siège de différences de phase importantes entre les deux voies microphoniques.

C'est pour cette raison que des vérifications périodiques en atelier ou chez le constructeur sont nécessaires.

$\left.4^{\circ}\right)$ Sonde appariée par conception:

L'avantage de cette technique est que la réponse en phase est assurée principalement par la sonde de sorte qu'il n'est plus nécessaire d'apparier les microphones en fréquence de coupure basse. Leurs dérives dans le temps deviennent donc insignifiantes ce qui rend inutiles les contrôles fréquents d'étalonnage.

Il ne faut toutefois pas en conclure que l'on peut aussi s'abstenir de vérifications périodiques car les membranes des microphones ne sont jamais à l'abri de microperforations par suite de corrosion.

$\left.5^{\circ}\right)$ Sonde symétrique.

L'une des méthode pour s'assurer la cohérence des mesures, préconisée par les normes ISO/DP 9614 et AFNOR S31-100 consiste à opérer un retournement de la sonde. Ce retournement est particulièrement simple à opérer si le centre acoustique de la sonde est sur l'axe de la poignée.

\section{SYNTHESES PRATIQUES}

Les sondes unidimensionnelles AKSUD, 9913 pour mesures de précision en laboratoire et, 9919 pour applications industrielles sont par conception appariées sur les deux voies et symétriques pour permettre l'inversion des voies par retournement. Les microphones sont équipés de grilles électrostatiques pour permettre un contrôle d'étalonnage simplifié sur site, sans démontage, à l'aide du calibreur 5699.

\section{ETALONNAGE SUR SITE ET EN LABORATOIRE}

L'étalonnage en laboratoire ou en atelier doit permettre de vérifier les caractéristiques essentielles indiquées sur la fiche technique délivrée avec la sonde.

Le contrôle d'étalonnage sur le site doit permettre de garantir la validité d'une série de mesures d'une manière simple et pratique, si possible sans démontage de la sonde.

C'est à cette fin qu'a été conçu le calibreur d'intensimétrie 5699 construit par AKSUD sous licence du CETIM.

En laboratoire ou en atelier, il permet l'étalonnage en phase de la sonde et de l'intensimètre, ainsi que l'étalonnage en amplitude des deux voies de mesure.

L'étalonnage en phase s'opère en cavité aux basses fréquences (entre $20 \mathrm{~Hz}$ et $1 \mathrm{kHz}$ ). II permet de déceler d'éventuelles microperforations des membranes des microphones si la fonction de transfert diffère notablement de celle contenue dans la fiche technique individuelle de la sonde.

Aux fréquences élevées, l'étalonnage en phase s'opère par excitation électrostatique des microphones.

Le 5699 dispose d'un générateur interne de bruit blanc ou rose pour l'étalonnage en phase, aussi bien en cavité qu'en excitation électrostatique. 
L'étalonnage en amplitude s'opère en cavité à l'aide du générateur interne à $250 \mathrm{~Hz}$ ou $1 \mathrm{kHz}$.

Sur le site, il suffit de vérifier l'étalonnage en phase par excitation électrostatique des microphones en bruit rose et de contrôler l'amplitude à $1 \mathrm{kHz}$. Ces opérations ne nécessitent aucun démontage des microphones.

\section{CONCLUSION}

Dans les applications industrielles de l'intensimétrie acoustique, le choix de la sonde est déterminant.

C'est pourquoi AKSUD propose l'association d'une sonde appariée par sa conception et symétrique par sa construction en vue de valider simplement une série de mesures selon les normes en vigueur.

AKSUD propose également un calibreur d'intensimétrie simple d'emploi pour les contrôles sur site, mais aussi adapté pour la vérification par l'utilisateur des principales caractéristiques de la sonde annoncées par le constructeur.

\section{REMERCIEMENTS}

Nous remercions particulièrement Monsieur T.M. PHAN du CETIM à Senlis, ainsi que Monsieur J.C. PATRAT du C.E.A.T. de Poitiers pour l'aide qu'ils nous ont apportée à la conception de nos sondes et de notre calibreur d'intensimétrie.

\section{BIBLIOGRAPHIE}

[1] J. WASMER : Différentes sources d'erreurs dans les mesures d'intensimétrie. 2ème Congrès Français d'acoustique - Arcachon 1992.

[2] P.V. BRÜEL : Configuration de microphones pour sondes d'intensitê acoustique. 2ème Congrès International sur l'intensimétrie acoustique - Senlis 1985.

[3] K. BELEFQIH et J.C. REBILLAT : Diffraction acoustique autour de sondes intensimétriques cylindriques. 2ème Congrès înternational sur l'intensimétrie acoustique - Senlis 1985.

[4] O. DYRLUND : A note on statistical errors in acoustic intensity measurements, 1983, Journal of sound and vibration, 90, pp. 585-589.

[5] E. FREDERIKSEN : Caractéristiques de phase des microphones destinés aux sondes d'intensité - 2ème Congrès acoustique sur l'intensimétrie acoustique - Senlis 1985.

[6] J.C. PATRAT et K. BELEFOIH : Intensimétrie acoustique à l'aide de matériel non spécialisé : problèmes d'étalonnage - 2ème Congrès acoustique sur l'intensimétrie - Senlis 1985. 